\title{
Relación entre las características socioeconómicas y el logro académico de los estudiantes en la primera infancia en Colombia $-2017-1$
}

DOI: https://doi.org/10.21158/23227230.v10.n0.2020.2945

\author{
Sara Sofía Caicedo-Reyes ${ }^{2}$ \\ Universidad Militar Nueva Granada \\ est.sara.caicedo@unimilitar.edu.co \\ Adriana Carolina Silva-Arias ${ }^{3}$ \\ Universidad Militar Nueva Granada \\ adriana.silva@unimilitar.edu.co
}

Cómo citar este artículo: Caicedo-Reyes, S. S.; Silva-Arias, A. C. (2020). Relación entre las características socioeconómicas y el logro académico de los estudiantes en la primera infancia en Colombia -2017-. Revista Ploutos, 10, (Páginas). DOI: https://doi.org/10.21158/23227230.v10.n0.2020.2945

Fecha de recepción: 02 de octubre de 2020

Fecha de aprobación: 19 de abril de 2021

\section{Resumen}

El principal objetivo de esta investigación fue analizar las características socioeconómicas y el logro educativo de los estudiantes de primaria en Colombia para el año 2017. Para ello se describe el logro educativo y algunas condiciones del contexto individual, familiar y de la institución educativa de los estudiantes. Este análisis es relevante, dado que las desigualdades en las oportunidades educativas inciden en la calidad de vida de los estudiantes, ya que, entre otras cosas, condicionan su nivel de ingreso y bienestar en la vida adulta. Así, los resultados evidenciaron que las circunstancias que se encuentran fuera del control de los estudiantes, como, por ejemplo, la condición socioeconómica, estuvieron asociadas a su desempeño académico. Igualmente, un entorno desfavorable individual, familiar y en los colegios se asoció con un menor logro educativo. Por tanto, este estudio contribuye a visibilizar que el sistema educativo reproduce las inequidades educativas y sociales desde la educación básica primaria en Colombia.

Palabras clave: desigualdad social; inequidad educativa; desempeño académico; rendimiento escolar; primera infancia.

\footnotetext{
1 Producto derivado del IMP ECO-3116 financiado por la Vicerrectoría de Investigaciones de la Universidad Militar Nueva Granada, Vigencia 2020-2021.

2 Estudiante del programa de Economía - Universidad Militar Nueva Granada. Integrante del Grupo de estudios de desigualdades sociales. ORCID: https://orcid.org/0000-0003-0043-0695

${ }^{3}$ Economista - Universidad del Rosario. Magíster en economía - Universidad de los Andes. Doctora en estudios de población - El Colegio de México. Líder del Grupo de estudios de desigualdades sociales y del Semillero de estudios económicos. ORCID: https://orcid.org/0000-0003-1610-881X
} 


\title{
Relationship between the socioeconomic characteristics and academic achievement of students in early childhood in Colombia -2017-
}

\begin{abstract}
The aim of this research was to analyze the socioeconomic characteristics and educational achievement of elementary school students in Colombia for the year 2017. For this purpose, we describe the educational achievement of the students, along with some of the conditions of the individual, their family, and their educational institution context. This analysis is relevant, given that inequalities in educational opportunities have an impact on the quality of life of students, and among other things, they condition their level of income and well-being in adult life. Thus, the results showed that circumstances beyond the students' control, such as their socioeconomic status, were associated with their academic performance. Likewise, unfavorable individual, family, and school environments were associated with lower educational achievement. Therefore, this study contributes to show that the educational system reproduces educational and social inequalities in Colombia from the very beginning, starting primary school.
\end{abstract}

Keywords: social inequality; educational inequity; academic achievement; school performance; early childhood.

Relação entre características socioeconômicas e desempenho acadêmico de alunos de primeira infância na Colômbia -2017-

\section{Resumo}

O objetivo principal desta pesquisa foi analisar as características socioeconômicas e o desempenho educacional dos alunos do ensino fundamental na Colômbia no ano de 2017. Para isso, são descritos o desempenho educacional e algumas condições do indivíduo, do contexto familiar e da instituição educacional dos alunos. Essa análise é relevante, já que as desigualdades nas oportunidades educacionais afetam a qualidade de vida dos alunos, uma vez que, entre outras coisas, condicionam seu nível de renda e bem-estar na vida adulta. Assim, os resultados mostraram que circunstâncias fora do controle dos alunos, como por exemplo a condição socioeconômica, estiveram associadas ao seu desempenho acadêmico. Da mesma forma, um ambiente desfavorável individual, familiar e escolar foi associado ao menor desempenho educacional. Portanto, este estudo contribui para tornar visível que o sistema educacional reproduz as desigualdades educacionais e sociais desde a educação básica na Colômbia.

Palavras-chave: desigualdade social, desigualdade educacional, desempenho acadêmico, rendimento escolar, primeira infância. 


\title{
Relations entre caractéristiques socio-économiques \\ et réussite scolaire des élèves en Colombie -2017-
}

\begin{abstract}
Résumé
Cette recherche analyse les caractéristiques socio-économiques et la réussite scolaire des élèves colombiens de primaire pour l'année 2017. L'article étudie à cette fin la réussite scolaire des collégiens et certaines conditions du contexte individuel, familial et de l'établissement d'enseignement. Cette analyse semble pertinente étant donné que les inégalités d'opportunités éducatives affectent la qualité de vie des élèves, conditionnant, entre autres, leur niveau de revenu futur et leur bien-être dans la vie adulte. Les résultats montrent ainsi que des circonstances indépendantes de la volonté des étudiants - comme le statut socio-économique familial - étaient associées aux résultats scolaires. De même, un environnement individuel, familial et scolaire défavorable est davantage propice à des résultats scolaires inférieurs. Cette étude contribue ainsi à rendre visible les inégalités éducatives et sociales du système éducatif colombien qui reproduit des inégalités sociétales fortement marquées.
\end{abstract}

Mots-clés: inégalité sociale; iniquité éducative; performance académique; résultats scolaire; petite enfance.

\section{Introducción}

El presente artículo de investigación analizó algunos factores asociados al logro académico de los estudiantes colombianos al finalizar la primaria en el 2017. Este análisis resulta importante, dado que las desigualdades se gestan en la educación de los estudiantes desde los primeros años de la trayectoria educativa.

El capital humano acumulado desde la infancia es una circunstancia que incide en las diferentes etapas que atraviesa el menor hasta llegar a su edad adulta (Ariza y Retajac, 2020; Munevar, Silva y Sarmiento, 2019; Veroux, 2020). Al respecto, son escasos los estudios que permiten evidenciar las desigualdades socioeconómicas al finalizar el primer ciclo educativo.

La literatura de desigualdad de oportunidades educativas evidencia que en el desempeño académico inciden variables tales como la habilidad personal, el contexto familiar y el ámbito institucional (Broer, Bai y Fonseca, 2019; Ferreira y Gignoux, 2011). En particular, la evidencia empírica sugiere una relación directa entre condiciones socioeconómicas y el desempeño académico (Ganimian y Murnane, 2016; Glewwe y Muralidharan, 2016). 
Adicionalmente, el enfoque en la educación básica es indispensable en razón al papel relevante de la educación temprana como factor indispensable con miras a igualar las oportunidades para el desarrollo de las personas y de la sociedad en su conjunto (Ferreira y Gignoux, 2014; Veroux, 2020). Por otra parte, las desigualdades sociales son un tema relevante para los hacedores de políticas públicas, ya que los condicionantes sociales de la primera infancia no deberían incidir en su desarrollo educativo (Hanushek y Woessmann, 2008; Österman, 2008).

Para llevar a cabo esta investigación, se hizo uso, principalmente, de la información del Censo de Educación Formal (EDUC) recopilada por el Departamento Administrativo Nacional de Estadística (DANE). Adicionalmente, se analiza la información del Instituto Colombiano para la Evaluación de la Educación (Icfes) de todos los estudiantes que presentaron las Pruebas Saber $5^{\circ}$ en el 2017. Estas fuentes de información permitieron evidenciar que un entorno desfavorable individual, familiar y en los colegios se asoció a un menor logro educativo.

Así, el presente documento se organiza en cuatro partes, siendo la primera la presente introducción. En la segunda sección se expone la metodología utilizada. Consecutivamente los resultados y, finalmente, se presentan las conclusiones.

\section{Metodología y datos}

Esta investigación se realizó a partir de la información del censo de educación formal (EDUC) realizado por el DANE. Además, se utilizó la información de las Pruebas Saber $5^{\circ}$ realizadas por el Icfes a los estudiantes de educación básica primaria en el 2017. El universo de estudio estuvo compuesto por los 597593 estudiantes de quinto de primaria que presentaron la prueba en el periodo de estudio.

El censo de educación formal (EDUC) recopila información de todas las sedes educativas que ofrecen los cuatro ciclos educativos o con modelos educativos flexibles en Colombia. Así, la información es de todas las sedes educativas públicas o privadas legalmente 
constituidas dentro del territorio nacional. Por otra parte, las pruebas Saber $5^{\circ}$ son realizadas a todos los estudiantes del territorio nacional que se encuentren dentro de una institución educativa formal, finalizando la educación primaria — en quinto grado - para evaluar las competencias básicas. De este modo, integramos la información de las sedes educativas y las características de los estudiantes con su desempeño académico en las pruebas estandarizadas.

Así mismo, esta investigación realizó un análisis descriptivo según la clasificación nivel socioeconómico categórico (NSE) brindada por el Icfes, ya que este indicador permite caracterizar la población que presenta las pruebas. Este indicador se calcula a partir del índice de nivel socioeconómico (INSE), estimado por el Icfes, el cual tienen en cuenta variables tales como posesión de bienes, acceso a servicios y educación del núcleo familiar. El nivel socioeconómico se agrupó en bajo y alto, siendo bajo para los estudiantes con NSE 1 o 2 y alto para los estudiantes con NSE 3 o 4.

Para llevar a cabo el análisis se consideraron variables que la literatura considera como factores asociados a las desigualdades en el logro educativo de los estudiantes. En primer lugar, el nivel educativo de los padres, debido a que se relaciona directamente con las aspiraciones y el logro educativo de los hijos. Así mismo, la disponibilidad de bienes de tecnologías de información y comunicaciones (TIC) porque influyen en la capacidad y facilidad de aprendizaje. En particular, se realizó un análisis descriptivo de las desigualdades en el logro educativo en lenguaje y matemáticas diferenciado por el contexto socioeconómico de los estudiantes.

\section{Resultados}

A partir de las consideraciones metodológicas enunciadas, esta sección compila el análisis descriptivo de la desigualdad de oportunidades en el logro educativo para los estudiantes de primaria en Colombia durante el 2017. En primer lugar, se tiene en cuenta que uno de los principales elementos que inciden en el logro educativo de los estudiantes es el capital físico y humano disponible en el hogar (Gamboa et al., 2003). 
$\mathrm{Al}$ respecto, en la figura 1 se evidencia que los estudiantes que tuvieron padres con bajo nivel educativo tuvieron, a la vez, bajo nivel socioeconómico. Estos estudiantes, además, presentaron un bajo logro académico. Lo anterior permite evidenciar que padres con un nivel académico más alto consideran importante la educación de sus hijos (Heineck y Riphahn, 2009; Veroux, 2020). Además, padres más escolarizados proporcionan mejores herramientas y mayor apoyo para el desarrollo oportuno de las actividades académicas (Ayala-García, Marrugo-Llorente y Saray-Ricardo, 2011; Husain et al., 2019).

Figura 1. Nivel educativo de los padres y nivel socioeconómico de los estudiantes de quinto primaria en Colombia, 2017

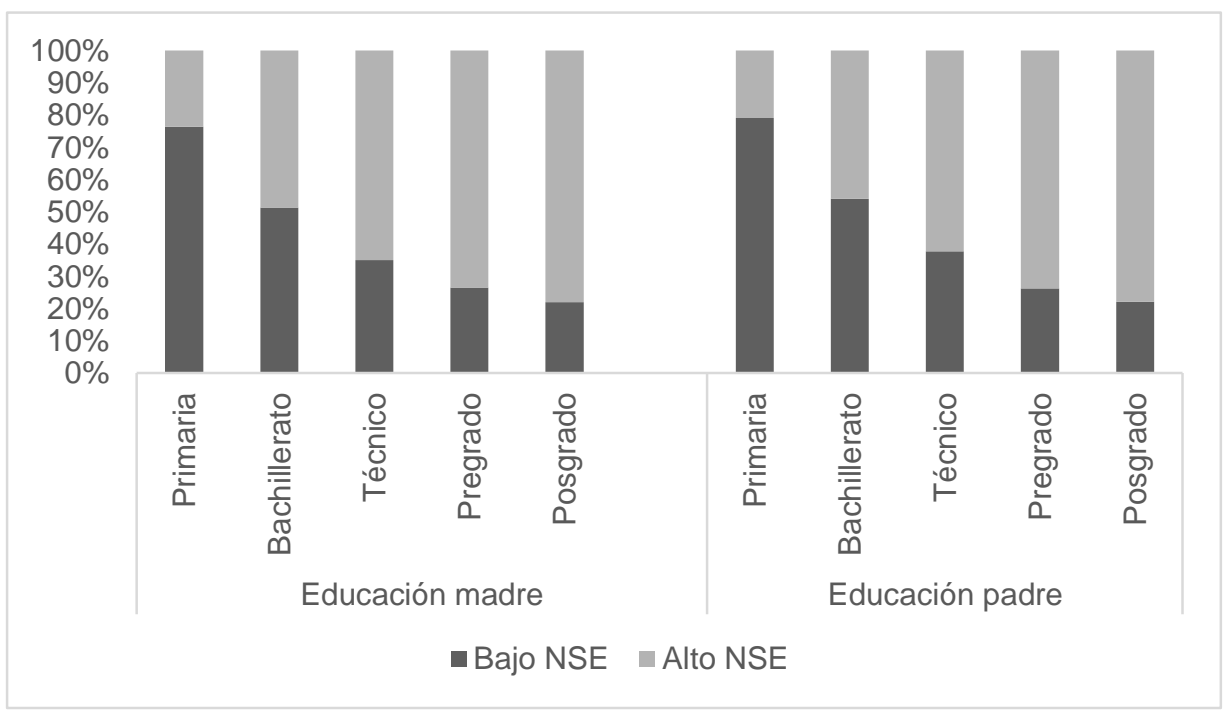

Fuente. Elaboración propia con base en datos del Icfes.

A continuación, se analizan otras circunstancias desfavorables que se encuentran fuera del control de los estudiantes y pudieron tener relación directa con características relacionadas con un menor logro educativo. En primer lugar, en la tabla 1 se evidencia que menos del $50 \%$ de los estudiantes con NSE bajo contaban con las herramientas tecnológicas básicas para desarrollar sus actividades académicas. En contraste con lo anterior, un poco menos del $15 \%$ de los individuos con NSE alto no tuvieron herramientas tecnológicas. 
En el contexto reciente del confinamiento debido a la contingencia sanitaria generada por el Covid-19, esta situación implicaría que más del $50 \%$ de los estudiantes de primaria en Colombia con NSE bajo no tendrían la posibilidad de tomar sus clases de forma remota, por lo que su logro académico podría ser aún menor. Por tanto, las desigualdades en el acceso a bienes y servicios TIC podrían estar asociados con mayores brechas educativas (Cruz, Hernández y Silva-Arias, 2020).

Tabla 1. Caracterización de los estudiantes de quinto de primaria en Colombia-2017

\begin{tabular}{|c|c|c|}
\hline & Bajo NSE & Alto NSE \\
\hline Cantidad de estudiantes & 379,344 & 218,249 \\
\hline \multicolumn{3}{|l|}{ Contexto familiar } \\
\hline \multicolumn{3}{|l|}{ ¿Tiene computador? } \\
\hline Sí & 0,477 & 0,849 \\
\hline No & 0,523 & 0,151 \\
\hline \multicolumn{3}{|l|}{ ¿Tiene internet? } \\
\hline Sí & 0,425 & 0,862 \\
\hline No & 0,575 & 0,138 \\
\hline \multicolumn{3}{|l|}{ Sector } \\
\hline No oficial & 0,049 & 0,502 \\
\hline Oficial & 0,951 & 0,498 \\
\hline \multicolumn{3}{|c|}{ Promedio Prueba Saber $5^{\circ}$} \\
\hline Lenguaje & 299,351 & 344,210 \\
\hline Matemáticas & 288,352 & 328,331 \\
\hline
\end{tabular}

Fuente. Elaboración propia con base en datos del Icfes.

En cuanto al sector de la institución educativa que atendió a los estudiantes por nivel socioeconómico, se evidencia que el $95 \%$ de los estudiantes con bajo NSE asistieron a colegios públicos. De otro lado, el 50 \% de los estudiantes con alto NSE asistieron a colegios privados. Por lo anterior, se evidenció que la mayor proporción de estudiantes con circunstancias desfavorables se encontraba en colegios públicos. Así, los esfuerzos en políticas públicas deberían enfocarse en fortalecer a las instituciones educativas públicas, en 
términos de equipos y conectividad, con el propósito de igualar las oportunidades educativas de los estudiantes con condiciones sociales adversas.

En cuanto al logro educativo, en general, se destaca que los estudiantes de quinto grado tuvieron mejor desempeño en lenguaje que en matemáticas. Respecto a las brechas educativas por circunstancias socioeconómicas, los puntajes promedio en las pruebas de lenguaje y matemáticas de los estudiantes con NSE bajo fueron de 299,351 y 288,352, respectivamente; mientras que los resultados promedio en lenguaje y matemáticas para los estudiantes con NSE alto fueron 344,21 y 328,331, respectivamente. De esta forma, se evidencia que los estudiantes con mayor nivel socioeconómico tuvieron un mucho mejor desempeño académico, considerando un rango de calificación de 0 a 500 puntos. La brecha en el logro educativo fue de alrededor de 40 puntos entre los resultados obtenidos por los estudiantes de uno y otro NSE.

En este sentido, la figura 2 muestra las brechas en el logro educativo en las pruebas de lenguaje y matemáticas entre los estudiantes del nivel socioeconómico bajo y alto. Igualmente, podemos resaltar el hecho de que un gran porcentaje de los resultados obtenidos por las personas de nivel socioeconómico bajo tuvo un desempeño insuficiente, mientras que los estudiantes del nivel socioeconómico más alto presentaron mayor prevalencia en el desempeño avanzado. 
Figura 2. Resultados por niveles en las pruebas de lenguaje y matemáticas de los estudiantes de quinto de primaria en Colombia, 2017

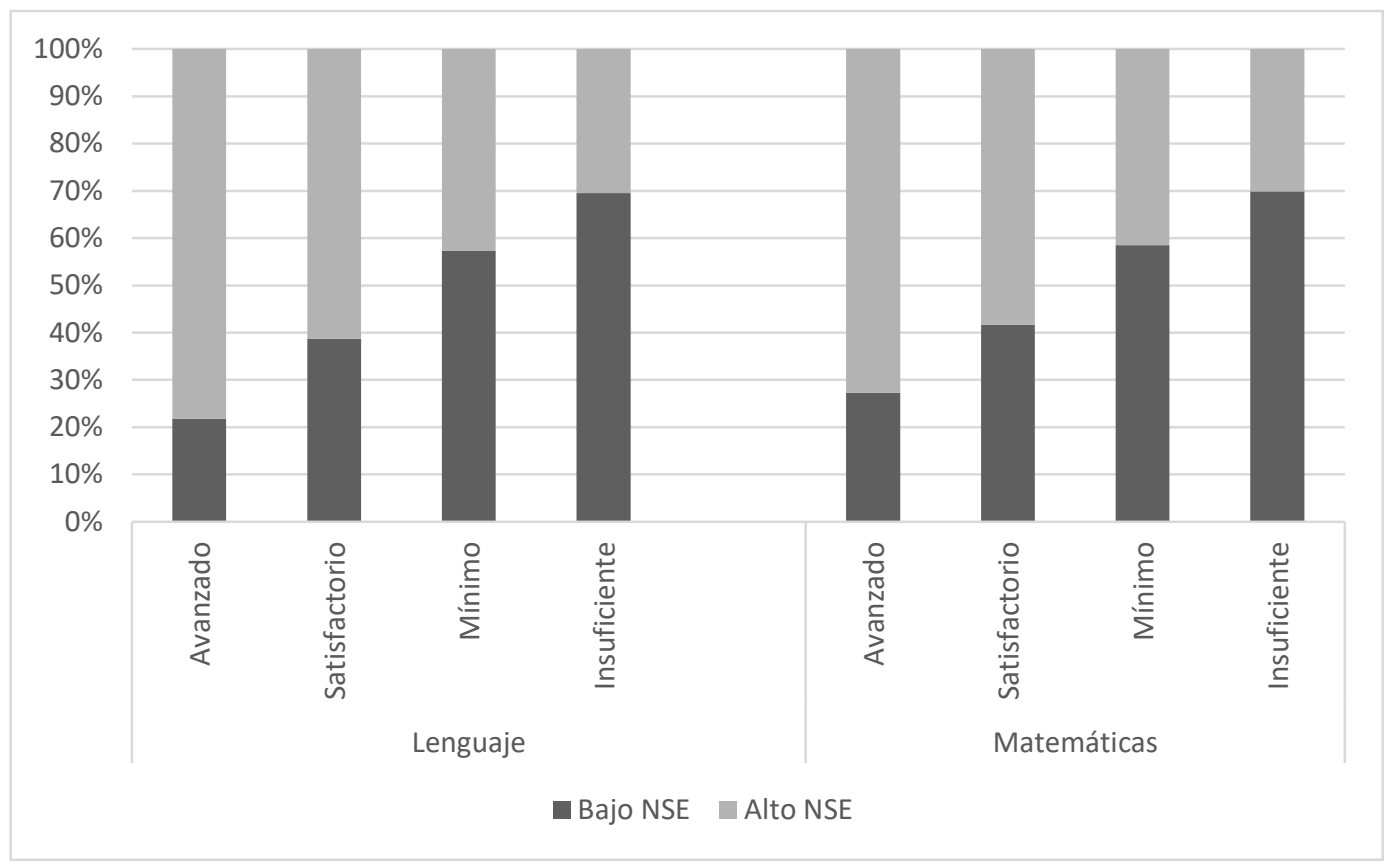

Fuente. Elaboración propia con datos del Icfes.

En síntesis, esta información muestra las inequidades sociales asociadas a las brechas educativas, por lo que resulta inapropiado caracterizar como deficientes a los estudiantes y a los colegios que no logran cierto logro educativo, ya que las circunstancias del contexto fueron distintas. Por tanto, en línea con la literatura de desigualdad de oportunidades educativas, los reportes del logro educativo en las pruebas estandarizadas deberían incluir un análisis de perfiles de estudiantes por circunstancias, a fin de poner de manifiesto que existen inequidades sociales que ahondan las inequidades educativas.

\section{Conclusiones}

La desigualdad de oportunidades educativas reproduce las diferencias sociales intergeneracionalmente, así como limita el crecimiento económico y el desarrollo (Paes de Barros et al., 2009; Hanushek y Woessmann, 2012; Marrero y Rodríguez, 2013). De esta forma, es relevante caracterizar las desigualdades en las circunstancias de los estudiantes y 
establecer cómo limitan su logro educativo desde los primeros ciclos de la trayectoria educativa. Este análisis descriptivo permite proporcionar instrumentos de política dirigidos a disminuir las brechas educativas, tales como la necesidad de incrementar los esfuerzos por dotar a los colegios públicos con los bienes TIC necesarios para promover un entorno académico que minimice las diferencias entre las herramientas y la infraestructura de los planteles con diferentes condiciones socioeconómicas.

En este artículo se evidencia la necesidad de no analizar comparativamente los resultados de aprendizaje de los estudiantes con menor nivel socioeconómico con los que obtuvieron los estudiantes con circunstancias más favorables, pues las brechas educativas estuvieron asociadas a las diferencias entre el entorno familiar y académico en el que se desenvolvieron los estudiantes de primaria en Colombia. De otro lado, al estudiar las brechas en el logro educativo, en aras de la equidad, deberían analizarse de acuerdo con las circunstancias que son ajenas a las habilidades y al esfuerzo del estudiante.

En cuanto a las políticas públicas que contribuyan a cerrar estas brechas, planteamos que la atención del primer ciclo educativo es primordial para esto. Por tanto, la calidad de la educación inicial no solo afecta la trayectoria educativa de los estudiantes, sino que se transmite a generaciones futuras. Por lo anterior, se requiere una política que mejore la calidad de la educación pública, en términos de herramientas que faciliten y promuevan el aprendizaje, tales como bienes TIC; la conformación del plan de estudios y la experticia de los docentes, dada su importancia e incidencia en el logro de los estudiantes (OCDE, et al., 2012; Hernández y Pérez, 2018; Kadir, 2019; Quintana-Torres, 2018). Además, teniendo en cuenta las limitaciones económicas que viven gran parte de las familias y los estudiantes que asisten a estos planteles, es primordial reducir las brechas que se profundizan y perpetúan en periodos de crisis. 


\section{Referencias}

Ariza, J.; Retajac, A. (2020). Impactos sociales de la educación (vol. 1). Ibagué: Sello Editorial Universidad del Tolima.

Ayala-García, J.; Marrugo-Llorente, S.; Saray-Ricardo, B. (2011). Antecedentes familiares y rendimiento académico en los colegios oficiales de Cartagena. Economía y Región, 5(2), 43-85. Recuperado de https://bit.ly/3yhZcet

Broer, M.; Bai, Y.; Fonseca, F. (2019). Socioeconomic inequality and educational outcomes: evidence from twenty years of TIMSS (vol. 5). Cham: Springer Nature. DOI: https://doi.org/10.1007/978-3-030-11991-1

Cruz, V.; Hernández, Á.; Silva-Arias, A. C. (2020). Cobertura de las TIC en la educación básica rural y urbana en Colombia. Revista Científica Profundidad Construyendo Futuro, 13(13), 39-48. DOI: https://doi.org/10.22463/24221783.2578

Ferreira, F.; Gignoux, J. (2011). The measurement of inequality of opportunity: theory and an application to Latin America. Review of Income and Wealth, 57(4), 622-657. DOI: https://doi.org/10.1111/i.1475-4991.2011.00467.x

Ferreira, F.; Gignoux, J. (2014). The measurement of educational inequality: achievement and opportunity. The World Bank Economic Review, 28(2), 210-246. DOI: https://doi.org/10.1093/wber/lht004

Gamboa, L.; Casas, A.; Piñeros, L. (2003). La teoría del valor agregado: una aproximación a la calidad de la educación en Colombia. Revista de Economía del Rosario, 6(2), 95-116. Recuperado de https://bit.ly/2TmLAzX

Ganimian, A.; Murnane, R. (2016). Improving education in developing countries: lessons from rigorous impact evaluations. Review of Educational Research, 86(3), 719-755. DOI: https://doi.org/10.3102/0034654315627499

Glewwe, P.; Muralidharan, K. (2016). Chapter 10-improving education outcomes in developing countries: evidence, knowledge gaps, and policy implications. En E. A. Hanushek; S. Machin; L. Woessmann (Eds.) Handbook of the economics of education. (Vol. 5, 653-743). Londres: Elsevier. DOI: https://doi.org/10.1016/B978-0-444-63459-7.00010-5

Hanushek, E. A.; Woessmann, L. (2008). The role of cognitive skills in economic development. Journal of Economic Literature, 46(3), 607-668. DOI: https://doi.org/10.1257/jel.46.3.607

Hanushek, E.; Woessmann, L. (2012). Do better schools lead to more growth? Cognitive skills, economic outcomes, and causation. Journal of Economic Growth, 17(4), 267-321. DOI: https://doi.org/10.1007/s10887-012-9081-x

Heineck, G.; Riphahn, R. (2009). Intergenerational transmission of educational attainment in Germany-the last five decades. Journal of Economics and Statistics, 229(1), 36-60. DOI: https://doi.org/10.1515/ibnst-2009-0104 
Hernández, F.; Pérez, M. (2018). Autonomía de gestión para la calidad y equidad educativa: una evaluación del Programa Escuelas de Calidad (PEC). Revista Mexicana de Análisis Político y Administración Pública, 7(2), 153-174.

Husain, N.; Meisenberg, G.; Becker, D.; Bakhiet, S.; Essa, Y., Lynn, R.; Al Julayghim, F. (2019). Intelligence, family income and parental education in the Sudan. Intelligence, 77, 101402. DOI: https://doi.org/10.1016/j.intell.2019.101402

Kadir, N. (2019). Management of secondary school teachers in enhancing quality education in Kwara State, Nigeria. Jurnal Pendidikan Malaysia, 44(2), 100-109. DOI: https://doi.org/10.17576/jpen-2019-44.02-10

Marrero, G. A.; Rodríguez, J. G. (2013). Inequality of opportunity and growth. Journal of Development Economics, 104, 107-122. DOI: https://doi.org/10.1016/j.jdeveco.2013.05.004

Munevar, S.; Silva, A.; Sarmiento, J. (2019). Exposición al conflicto armado y logro académico en Colombia. Revista Desarrollo y Sociedad, 83, 13-53. DOI: https://doi.org/10.13043/dys.83.1

OCDE; Banco Internacional de Reconstrucción y Fomento; Banco Mundial. (2012). Evaluaciones de políticas nacionales de educación: la educación superior en Colombia. París: OCDE Publishing. DOI: https://doi.org/10.1787/9789264180710-es

Österman, M. (2018). Varieties of education and inequality: how the institutions of education and political economy condition inequality. Socio-Economic Review, 16(1), 113-135. DOI: https://doi.org/10.1093/ser/mwx007

Paes de Barros, R. de; Ferreira, F. H. G.; Molinas-Vega, J.; Saavedra-Chanduvi, J. (2009). Measuring inequality of opportunities in Latin America and the Caribbean. Washington: The World Bank.

Quintana-Torres, Y. (2018). Calidad educativa y gestión escolar: una relación dinámica. Educación y Educadores, 21(2), 259-281. DOI: https://doi.org/10.5294/edu.2018.21.2.5

Veroux, O. A. (2020). Trends of intergenerational transmission of education. Evidence from Colombia (Tesis de maestría). Lund University. Lund, Suecia. Recuperado de http://lup.lub.lu.se/student-papers/record/9021134 